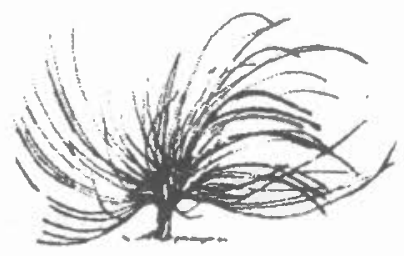

\title{
Mis vivencias hacia el camino del maestro
}

\author{
Enrique Vilchez Quesadal \\ Universidad Nacional, Costa Rica \\ Heredia, Costa Rica \\ evq1529@gmail.com
}

\section{Resumen}

Esta biografia trata de mis vivencias hacia el camino de maestro, de los principios básicos del aprendiz tras años de formación, memorias de mi primer maestro, las enseñanzas del mejor profesor. En este recorrido arduo se forjan cimientos culturales y académicos que, diluidos en las mentes soñadoras, se transforman en expectativas que determinan nuestros ideales humanos.

Palabras claves: vivencias, aprendiz, elocuente profesor, discípulo, enseñanza, labor educativa, sociedad, profesional, académico.

\begin{abstract}
This is a biography about my experience in the way of becoming a teacher, about the basic principles of the apprentice after years of experience, about the memories of my first teacher and about the teachings of my best professor. In this tough journey, many academic and cultural basis are forged which, diluted in dreaming minds, transform themselves in order to determine our human ideals.
\end{abstract}

Recibido: 08 de setiembre, 2010 - Aprobado: 29 de junio, 2011

1 Magister y profesor de la Escuela de Informática de la Universidad Nacional. 
Keywords: Experiences, apprentice, disciple, teaching, educational work, society, professional, academic, eloquent teacher.

$\mathrm{H}$ ace ya bastantes años inicié con gran júbilo mi labor educativa. Al egresarme de la universidad mi principal objetivo como un joven profesionista, residió en contribuir con la sociedad hacia la construcción de futuro, no el mío naturalmente, sino el de los demás. Esta a mi juicio es la tarea más importante que circunscribe cualquier acto educativo, pensar siempre en los demás en un camino que en el mejor de los casos nos deriva a la alteridad y a la benevolencia. Claro está, este camino combina empirismo y teoria muchas veces ocultados bajo el desosiego de la cotidianidad y el dogmatismo del éxito social.

Cuando se inicia la vida profesional en la enseñanza, los principios básicos del neófito aprendiz se remontan a todo lo que la universidad pudo transmitirnos tras años de formación. En este arduo recorrido se forjan cimientos culturales y académicos que diluidos en las mentes soñadoras, se transforman en expectativas que determinan nuestros ideales humanos. En este sentido, ¿cuántos de nosotros no añorábamos en nuestra época universitaria, saber tanto como aquel elocuente profesor?, o, ¿evocar en nuestros estudiantes un interés tal, como lo hacía aquel destacado maestro, donde a nadie aburría y todos con ilusión escuchaban su amplia doctrina? Yo mismo recuerdo en esta época ya bastante arcaica en mi memoria, las enseñanzas del mejor profesor que tenía la Facultad, admiraba su forma pausada de hablar, su aspecto impecable frente al salón de clase, su dominio magistral de los temas a enseñar y su típica humildad combinada con un pronunciado recelo, por el alto valor que daba a su labor docente. Ya para entonces, mi floreciente idealismo juvenil, venía exigiendo la cautivante visión de un maestro verdadero, por quien durante muchos años profesé una esculpida vocación. Una de mis metas era llegar a ser como él. Durante años trabajé en ello, copie su modo de enseñar e inclusive su forma de escritura, aunque a ciencia cierta, nunca logré trazar con la misma precisión y belleza caligráfica, mis notas de clase en el pizarrón.

Conviene en este punto aclarar la importancia del término que he acuñado como maestro. En muchos contextos la palabra maestro es utilizada a la ligera como un simple sinónimo de profesor. En mi caso concibo que un maestro tiene un significado más legendario y lo 
relaciono directamente con el término chino shifu. En la tradición china de las artes marciales, un discípulo llamaba a su maestro shifu, shi podría traducirse como profesor y fu como padre. Bajo esta perspectiva, un maestro no solamente enseña lo que implica su competencia, enseña como un padre a su aprendiz, trascendiendo la obligatoriedad a una entrega total, donde no hay límites de tiempo y espacio.

Retomando mis memorias de mi primer maestro verdadero, recuerdo que durante este proceso mi ansiedad esotérica por seguir sus enseñanzas, dejó una huella muy profunda, delineando a su paso lo que para mí como educador significaba construir futuro. En principio consideré indispensable el dominio del conocimiento científico como fuente principal para el perfeccionamiento del pensamiento y la conciencia. Mi visión sin darme cuenta en ese entonces, se centraba en el positivismo y en una concepción dogmática acerca de la posibilidad del conocimiento. Bajo esta perspectiva filosófica, se asume que la transferencia de las propiedades del objeto que se aprende al sujeto que lo estudia, son posibles a través del uso de la razón, este enfoque por tanto es logicista e ignora cuestiones tales como: las ideas preconcebidas de la conciencia, la espontaneidad del sujeto y la complejidad de los criterios de verdad en los procesos del conocimiento. Dicha concepción propia de las ciencias exactas, reinó durante muchos años lo que podría llamar mi visión prehistórica de la enseñanza y el aprendizaje.

Pese a ello, esto es lo que más me había reconfortado en mis años universitarios, saberme poseedor del conocimiento y más aún, encontrarme a mí mismo con cierto grado de habilidad en el auto conocimiento. Auto conocimiento significa metacognición, es decir, el pensamiento sobre el pensamiento, un proceso que involucra el análisis sobre nuestros propios constructos de razonamiento. Lamentablemente durante esta eufórica etapa, mis ideales por el conocimiento con un fin en sí mismo, se hicieron palpables en mis primeras experiencias de enseñanza.

La primera vez que me enfrenté a un grupo de estudiantes en un salón de clase, lo hice en el contexto de un colegio nocturno en la provincia de Heredia ${ }^{2}$, donde al parecer las flores se marchitan al ocultarse el sol. Un viejo amigo quien en mis años de formación secundaria me había impartido clases particulares de matemática, accedió en calidad

2 Esta provincia se conoce en Costa Rica como la ciudad de las flores ... los abuelos cuentan: "por sus bellas mujeres". 
de préstamo, ofrecerme la oportunidad de aprender a ser docente en la lúdica de la prueba y el error, a través de un grupo de octavo nivel con quien realizaría mi práctica profesional. El primer día me sentía tan nervioso al punto de cuestionar la selección que había hecho de mi carrera al ingresar en la universidad. Cuando llegué al salón de clase, curiosamente en el aula asignada no había corriente eléctrica, lo más extraño del suceso era que la corriente se ausentaba única y exclusivamente donde me correspondía impartir mi primera lección. Pregunté a los estudiantes qué había ocurrido y con una bruñida mirada ¡nadie sabía nada al respecto! Me sentía muy frustrado pues jera mi primera lección! y ya me encontraba retrasado sin saber que hacer ... dentro del salón del clase no se veía absolutamente nada, algo así como la casa de los sustos que me ocasionaba pesadillas en mi infancia. Poco después llego el dueño y señor de la clase -mi estimado amigo- sin preguntar a nadie levantó la cuchilla de la corriente y por arte de magia todo volvió a su normalidad, las risas en el pasillo todavía hacen eco en mis memorables recuerdos.

Ese semestre fue uno de los más largos y agotadores períodos que ha esa fecha había vivido, mis ideas preconcebidas respecto al valor fundamental del conocimiento se comenzaban a desmoronar. Mis lecciones catedráticas constantemente aburrían a los estudiantes, quienes al estar matriculados en un colegio nocturno, en su mayoría tenían que laborar fuertes jornadas diurnas para finalizar felizmente su apatía, con un "tipo" que les hablaba en otro idioma. Desde luego, imagínense las caras de aquellos estudiantes ... yo me sentía libre al fin de dictar mis amplios conocimientos en la materia, nadie podía impedírmelo, era mi gran oportunidad de demostrar todo lo que la universidad me había enseñado ... En una de estas desconcertantes lecciones, recuerdo a un estudiante levantar la mano, yo desde luego sentia la necesidad de continuar ... de lo contrario no podría cumplir con lo programado para esa fecha. Dos voces en mi interior parecían hablarme al mismo tiempo:

-Hey, hey ... me escuchas ... aquel estudiante en la esquina del fondo está levantando la mano, ¿no vas a cederle la palabra? De pronto irrumpió un segundo personaje en mi conciencia:

-Vamos, vamos, si les das la palabra ... jno vas a terminar los contenidos que tenías que cubrir para hoy! El dilema continuó y al término de una confusa discusión, regresé de este corto viaje y decidí escuchar lo que aquel estudiante tenía tantas ganas de decir, dado que pese a diez minutos de indiferencia, el alumno insistía con su mano levantada: 
-Profesor -dijo- sus explicaciones son muy enredadas y no entiendo nada ... Sabe, hoy me dieron un revolver en mi trabajo ... Soy guarda -replicó con gran orgullo- ¿quiere verla? Y de pronto extrajo de su bolso escolar una pistola calibre 22. Entré en shock pues nunca antes en mi vida -para entonces tenía 20 años de edad- había mirado un arma en vivo y a todo color. Toda el aula se levantó corriendo a admirar la noble causa que los había sacado de la tortura. Esa fue mi primera amarga lección, respecto al engaño que yo mismo había forjado con relación al camino hacia al maestro. Mis creencias estaban ahora al borde del precipicio... ¡no estaba construyendo futuro!

Años después, ya habiendo obtenido un título universitario, comencé con gran ilusión mi carrera profesional. Laboraba para el Ministerio de Educación Pública y había obtenido "una plaza". Un puesto que se le da a un docente en una institución de enseñanza primaria o secundaria, interpretado muchas veces como una licencia laboral de "soy inamobible". Será por ello que en la actualidad las políticas de gobierno están orientadas a eliminar este tipo de "posibilidades sin fin".

Inicié mi tarea docente con tres grupos de décimo año, mi inspiración llegaba nuevamente a un punto de ebullición. Comencé la experiencia educativa como solía hacerlo, tenía que demostrar todos los conocimientos que poseia, este era el punto de partida para hacer la diferencia entre "el dios del Olimpo" y sus súbditos. Lo que aún no sabía este dios mitológico nuevo en el vecindario, era que en aquel reino, los súbditos con suma frecuencia solían amotinarse en contra de quienes eliminaban su zona de confort y que al dios de dioses que algunos miembros del consulado llamaban director, le agradaba quedar bien con el jurado popular, los padres de familia.

Con mucha inocencia nada de esto se hizo evidente al principio, mis clases nuevamente con fuertes características magistrales, alababan más el valor del conocimiento que el valor de la formación. Al finalizar mi primera prueba parcial, solamente un estudiante había superado con éxito tal reto. Los demás alumnos se encontraban en una situación preocupante que con naturalidad transfirieron a sus padres. En pocos días los malestares se hicieron sentir con mucha rapidez, el director, el supervisor, el asesor regional, el asesor nacional y el asesor de evaluación, reunieron todas sus tropas de apoyo en la institución para exigir explicaciones y calmar las amotinadas masas que buscaban un camino fácil frente a la actitud de un docente inflexible. 
Pocos días después, sin haber llegado a una solución conjunta, pues todos creían aún tener la única verdad, ocurrió en la institución una de las experiencias más impactantes que hasta ese momento se habian vivido dentro del sistema educativo nacional. Un alumno de décimo año había tomado prestada el arma de fuego de su padre. Ese día temprano en la mañana y con la ventaja de la ausencia de un adulto (aspecto característico de este hogar), el joven observó el arma y sin dudarlo un segundo pensó en la posibilidad de asombrar a sus compañeros de clase con esta adquisición. Las horas transcurrieron y en un inocente forcejeo durante el tiempo de recreo, el arma se disparó accidentalmente. El hecho fue tan rápido que al principio parecía solamente una travesura ... poco después se descubrió a una estudiante de primer año herida frente a la plaza deportiva. Sus compañeros gritaban y los docentes corrieron en su auxilio. $\mathrm{La}$ bala había atravesado su pantorrilla derecha, la niña se había trasladado a la dirección escolar y con mucha prontitud tanto la policía como los paramédicos estaban ya presentes, sin darnos cuenta inclusive los medios de comunicación masiva esperan afuera de las instalaciones con el principal objetivo de ganarse la premiere de la noticia del momento.

Con gran consternación el estudiante que había causado de este trágico acontecimiento, me buscó en el aula de clase con gran nerviosismo y lágrimas en sus ojos. Poco a poco comenzó a describirme todo lo sucedido pues yo era su "profesor guía". Aparentemente una de las tareas de los profesores guías en el sistema de educación pública de Costa Rica, consiste en atender individualmente a los alumnos para conocer sus aflicciones y sanar sus heridas psicológicas, algo para lo cual la universidad nunca me había preparado. Atónito escuché las palabras del efebo y sin saber exactamente cómo, la intuición me condujo a tranquilizar el dolor y la preocupación de aquel estudiante. Pronto me encontraba en la dirección, la niña herida sangraba sin cesar y el director de la institución me miraba pálido sin decir palabra alguna... he de imaginar que lo único que cruzaba por su mente, eran el conjunto de explicaciones que podría dar a los medios de comunicación, sin que ellos a su paso, en las tareas de post edición, trastornaran sus declaraciones. Fue una semana muy dificil... al término, afortunadamente la niña con la rehabilitación médica adecuada se recuperaría sin problemas. Mi estudiante por el contrario... fue expulsado del colegio y a la fecha lo recuerdo y me pregunto, si este joven como muchos, fue una víctima o un culpable. 
En la actualidad los actos de violencia en las instituciones de enseñanza en Costa Rica, constituyen uno de los problemas sociales más serios que enfrenta el Ministerio de Educación Pública y la sociedad en general. Hace pocos meses la línea que dividía la autoridad de la indisciplina y el liderazgo de la incomprensión se transgredió, aparentemente un joven estudiante de una institución educativa, molesto por las constantes reprimendas de la directora, consideró necesario tomar venganza... al igual que mi joven aprendiz, por quien no pude hacer nada, este alumno tomó prestada el arma de su padre y disparó. El suceso culminó con la muerte de la directora y el sufrimiento nacional ... por la impotencia, por la desesperanza, por el horror de hacernos cada uno de nosotros responsables de un acto que representa el corazón colectivo de una sociedad que margina al marginado, empobrece al empobrecido y humilla al humillado.

En el camino hacia el maestro, comprendí entonces que mi labor educativa muchas veces residía en prestar mi hombro para consolar y extender mi brazo para sosegar la angustia y la desesperación. Con el pasar del tiempo, inicié un proceso de transformación donde aquel conjunto de teorías del aprendizaje que había estudiado con detenimiento en la universidad, cobraban vida en un contexto que superaba el plano de la ciencia, un contexto netamente humano, donde el objetivo principal es ayudar a otros a entender cómo alcanzar la felicidad y la paz. Hoy por hoy, no logro comprender por qué en muchos aspectos la ciencia se separa del conocimiento profundo del alma.

Aprender a conocer el alma duele, pues muchas veces el alma solo se transforma después del sufrimiento. Este encuentro... dio inicio a una nueva etapa de mi labor educativa que podría llamar una visión "anam cara" de la enseñanza y el aprendizaje. El término anam cara que rescaté del libro "Sabiduría Celta” de John O'Donohue, significa "amigo del alma".

Como docentes en muchos aspectos seamos conscientes de ello o no, nos convertimos en amigos del alma, dado que conformamos modelos a seguir por parte de nuestros estudiantes. Los alumnos observan nuestra forma de vestir, nuestra forma de hablar y más aún, nuestra forma de comportarnos. Somos entonces de alguna manera, responsables del comportamiento que los estudiantes asumen en sus propias vidas. Esta postura un tanto agnóstica, nos aproxima al conocido eslogan "debemos predicar con nuestro ejemplo". 
Como consecuencia de esta refiexión, recuerdo un simple acontecimiento que me ha acompañado durante varias generaciones. Cuando cursaba el quinto año de la educación secundaria, un grupo de compañeros y este servidor, nos encontrábamos en la entrada del colegio. Juntos conversábamos con gracia sobre nuestros asuntos: juegos de video, remedábamos al profesor más duro del colegio y chismorreábamos las relaciones amorosas que todos imaginaban el director mantenía con la profesora de matemáticas. Al instante topamos con el profesor de filosofia, un tipo alto, de tez muy blanca pero que siempre mantenía en sus mejillas un extraño color rosa. Este personaje se había ganado la fama institucional, de favorecer largas horas a su hígado ingiriendo grandes cantidades de alcohol. Yo personalmente nunca había creído tal acusación, me parecía una persona chistosa, agradable y de buen ánimo con los estudiantes. El docente al pasar junto a nosotros, nos saludó con una pícara sonrisa, alguien dijo: ¡Qué profe!, ¿va para la choza?, en nuestra época estudiantil se utilizaba de una manera más o menos agreste, el término "choza" para referirse a una casa habitacional. El docente con su sonrisa aún más pronunciada, respondió: ¡Sí y sino al frente! Bueno, para quienes no conozcan la provincia de Heredia, nuestro querido filósofo había entendido que al hablar de la "choza" nos referíamos a un bar en las inmediaciones de la provincia que tenía este mismo nombre y que por cierto al frente de él, existía otro establecimiento de la misma naturaleza. Este hecho se quedó conmigo hasta mi vida adulta, fue tal el impacto de aquella confirmación que nunca lo pude olvidar. Ciertamente al finalizar el año escolar, algunos de nosotros visitamos este bar ... la aventura del absurdo intrinseca en la mente adolescente, nos condujo a seguir un mal ejemplo.

De nuevo en mis años como docente de enseñanza media y bajo el estandarte de una nueva concepción de la tarea educativa, inicié una reveladora búsqueda de almas necesitadas de un anam cara. Con prontitud me percaté de mi imposibilidad humana y profesional de ayudar a todos los que requerían de este auxilio, la demanda era muy alta y mis posibilidades muy escasas. Decidí entonces comenzar a seleccionar.

Mi primera aventura de rescate espiritual, la encontré en un alumno de sétimo año muy introvertido. Su historia familiar traslucía constantes agresiones físicas y un maltrato que ya había afectado su comportamiento natural. El alumno hablaba muy poco y a cualquier indicación dentro del salón de clase reaccionaba con un constante temor. 
Logré durante los primeros meses del año escolar, que este estudiante sitiera la confianza para sentarse junto a mi escritorio. Nuestra relación alumno-maestro poco a poco sentó las bases de una sólida amistad, que se fue transformando en el apoyo que un padre trata de dar a un hijo. Me di cuenta de las extraordinarias habilidades intelectuales que poseía sin estímulo alguno, más que la intervención divina que suele en algunas ocasiones favorecer al menos favorecido. Su habilidad en el razonamiento lógico me impresionó y como un proyecto personal inicié una etapa de formación en la que pude participar durante tres años consecutivos hasta el noveno grado. Esta mente brillante logró convertirse después de varios años de esfuerzo en un ingeniero civil, pese a todos los pronósticos dadas sus circunstancias familiares y económicas.

El recuerdo me sensibiliza a pensar en la labor educativa de miles de anam caras absorbidos por el anonimato de la cotidianidad y la perseverancia de sus principios morales y profesionales, quienes en su responsable labor educativa ofrecen nuevas oportunidades de crecimiento integral a cientos y cientos de jóvenes que luchan por sobrevivir situaciones de adversidad, que sólo a través de un "amigo del alma" podrán superar.

Ciertamente el camino hacia el maestro, también me ha hecho traspasar las barreras de la impotencia y el desconsuelo. En la dura tarea de la formación, que entiendo en mi actual sumatoria de experiencias de vida, como un altruismo personalizado en la construcción de futuro, muchas veces el acto de la corrección es interpretado como una provocación de los más bajos instintos humanos. Randy Pausch profesor de la prestigiosa universidad Carnegie Mellon, en su libro "La última lección", describe el valor de la autoestima como algo que los jóvenes deben construir con su esfuerzo. La labor del maestro no es dar autoestima, consiste en facilitar de forma imprescindible distintos momentos para el error y la corrección, un error bien aprendido puede contribuir directamente con el desarrollo de la autoestima. Cuando un docente renuncia a la corrección, renuncia al mismo tiempo a la formación de sus estudiantes. En el peor de los casos, el señalamiento del error muchas veces puede hacer emerger situaciones de competencia de poder, que desembocan en actos de venganza. Recuerdo hace algunos años, no tan lejanos ... los bajos resultados que un grupo de estudiantes habían obtenido en un examen parcial, era la primera vez que un profesor en una prueba de tal naturaleza, los había calificado así. Poco después, estos 
alumnos sin haber logrado entender mis argumentos en el ejercicio de la corrección, castigaron mi viejo automóvil en el estacionamiento de aquel lugar. Dos rayones cruzaban de lado a lado la tapa principal que cubría el motor. Cuando miré tal acto vandálico, sentí una sensación de impotencia muy profunda y mi corazón ya un tanto trastabillado por las circunstancias de la vida, se sitió muy herido y desconsolado pues sabía en la profundidad de mi conciencia, que aquello había sido el irónico resultado de mi interés por mis estudiantes. Nunca busqué pruebas para denunciar a aquel grupo de aprendices y durante ese semestre, el carro siguió sufriendo las consecuencias de mi resistencia. Al cabo del periodo, la ingratitud de un trabajo bien logrado, me condujo al riesgo de renunciar... ¿Cuántos educadores y educadoras a lo largo del territorio nacional, se han encontrado en una situación similar o de mayor riego inclusive en su propia integridad física?, el camino hacia el maestro por tanto, adicionalmente es un camino hacia la fortaleza y la valentía.

El error y la distorsión también han tomado parte en el recorrido de la formación sin fin hacia el significado profundo y real de la tarea educativa. La sociedad en general ha sido testigo de este conjunto de distorsiones, que han llevado inclusive, a ex presidentes de la república de nuestro país, a encontrarse encarcelados por sus súbitas inclinaciones hacia la ambición. Inclinaciones por cierto, que a mi juicio como seres humanos, todos tenemos. La diferencia radica en el ámbito de acción que nos mueve a dejar caer o no nuestros principios éticos y morales. Hace algunos años conocí a un colega de departamento en una institución, este jovial personaje fue bendecido con el don de la simpatía. Cualquier cosa que argumentaba, se hacía acompañar de pintorescos comentarios que con una impresionante afabilidad transformaban un insulto en un cumplido. Durante algún tiempo nuestra relación profesional fue un poco más cercana y en algún momento ya podía ser considerada una reciente amistad. Mi nuevo amigo comenzó a compartir sus experiencias de vida que pronto me comenzaron a abrumar. Su estilo de vida se hacía palpable en la manera en cómo los estudiantes estructuraban una visión de mundo donde todo tenía un precio. Consideré que su posición era un tanto ajena a mis propios principios éticos, sin querer decir con ello, que en mi vida no haya cometido errores como cualquier otro. La consternación de sus confesiones, me orilló a conservar única y exclusivamente nuestra vinculación profesional. 
Al finalizar el año lectivo lo visitaba con frecuencia en su salón de clase, nos gustaba discutir soluciones a diversos problemas de la disciplina y del mismo modo aspectos relacionados con la promoción estudiantil del departamento. Ya existía la confianza de correr la puerta y entrar evitando los formalismos y las reglas de etiqueta. En una de mis visitas, corrí la puerta del aula como era mi mala costumbre y en el acto, mi colega y una alumna que se encontraba con él, parecían un tanto sospechosos, mi presencia no produjo ninguna razón de preocupación y prosiguieron con sus "negocios", la alumna le entregaba a su profesor una calculadora y dentro de ella el pase mágico al próximo nivel. La estudiante llevaba rayada la materia durante todos los trimestres y no sentía una posibilidad real de aprobar el examen comprensivo de convocatoria. Mi estimado compañero de departamento ya entregado en un abismo sin retroceso, me miro con una sonrisa complaciente por haber logrado sellar con éxito su transacción. Nunca tuve pruebas suficientes que me dieran el valor para denunciar tal acto, sin embargo, cuando se cruza la línea de la cordura, la razón misma se convierte en juez y jurado. Mi imprudente compañero fue despedido años más tarde, la continuidad de sus actividades ilícitas que con los años producen el riesgo de la excesiva confianza, lo condujeron a una investigación interna que se resolvió fácilmente.

Estas breves reflexiones y recuerdos constituyen a mi juicio representaciones colectivas de problemas y hechos compartidos por los docentes en cualquier nivel en las instituciones educativas en Costa Rica. Mis simples vivencias procuran transmitir la apoteosis existencial en la cual con gran regocijo, se ha consolidado mi búsqueda insaciable hacia el camino de un verdadero maestro. Esta búsqueda me ha hecho vivenciar el resultado de una evolución continua de las teorías del aprendizaje en mi propia práctica educativa. Un recorrido cronológico que como ya lo he descrito, se remonta desde la praxis de un conductismo extremo hasta una visión más integradora a través del constructivismo social, con el que actualmente nutro mis nuevas vivencias.

Quisiera sistematizar de alguna forma las ideas que he expuesto en los párrafos anteriores. La historia de la investigación científica en el aprendizaje y el conocimiento de la mente humana parece trazar un claro camino que en un ejercicio introspectivo nos podría ocasionar un cierto recelo por la marcada brecha existente entre la teoría y las prácticas educativas en las instituciones escolares. La visión positivista 
del conocimiento en las escuelas (mi antigua visión pre histórica de la enseñanza y el aprendizaje) y más allá de ella en los planes, programas de estudio y políticas educativas, evidencia una acepción del aprendizaje no como un constructo que se desarrolla por medio de la guía, mediación y monitoreo del maestro, sino más bien, como un ente homogéneo desvinculado de la realidad del aprendiz, aislado de las otras disciplinas y transitorio, pues esta fragmentación ocasiona en el alumnado un conocimiento superficial que fácilmente se olvida detrás de las pruebas de cada período.

Reflexionar acerca de la enseñanza como lo he pretendido hacer en esta autobiografia, necesariamente nos obliga a confrontar tanto en la práctica como en la teoría, nuestras concepciones como docentes acerca del aprendizaje. Comúnmente en las instituciones de educación en cualquier nivel, el aprendizaje es concebido como consecuencia de la enseñanza o instrucción, sin embargo, al replantearnos tal situación, la teoría cognitiva y la tendencia empirica, parece sugerimos una postura opuesta, el aprendizaje se convierte entonces en la variable independiente que condiciona o determina a la enseñanza.

En este sentido, esta auto reflexión nos implica un reto complejo: las ideas, creencias, experiencias y conocimientos preconcebidos en nuestros estudiantes, tienen una relación directa con el aprendizaje hacia la comprensión y más aún con el aprendizaje trasferible hacia otros contextos y problemas, ignorar esta premisa, nos conduciría a la falacia de juzgar nuestro conocimiento y visión de mundo, como un objeto o paquete de información transferible. No somos como educadores, conductores de conocimiento, somos facilitadores de desarrollo de este conocimiento en un proceso que nos demanda una formación teórica fundamentada de contenido y en sus posibilidades de transferencia práctica en la resolución de problemas científicos e interpersonales. Sumado a ello se encuentra el valor del desarrollo de habilidades metacognitivas, es decir, habilidades que el estudiante utilice para comprender sus formas de pensamiento, sus sentimientos, su ser y sus propias estrategias de aprendizaje formal e informal.

Estos planteamientos sólidamente justificados en la investigación educativa, parecen en muchas instituciones de enseñanza irreconciliables con las prácticas docentes asumidas en el currículum oculto. Las razones de ello pbedecen desde mi perspectiva a diversas fuentes: la preparación positivista que reciben los futuros educadores o investigadores en 
ciencias sociales, las características de un sistema educativo centralizado en los gobiernos y las decisiones de escritorio y, la ausencia de una conceptualización clara de principios educativos que respondan al qué, por qué y para qué de la enseñanza.

En muchas universidades se aborda la formación en ciencias de la educación bajo una perspectiva eminentemente teórica, tal es el extremo de este enfoque formativo, que cuando los futuros docentes realizan su práctica de campo, muchas veces topan con la sorpresa de realidades totalmente dispares con lo aprendido en las aulas universitarias (ya describí en mi propio contexto esta frustrante vivencia). El choque teoríapráctica parece disolver la esperanza de los neófitos educadores en la tradición de los sistemas educativos que apuntan al cumplimiento de temarios y no a la comprensión profunda de los conocimientos teóricos y existenciales que se espera difuminar en la nuevas generaciones. Por ello, resulta interesante hacer notar, que si como docentes pretendemos enseñar a nuestros estudiantes estrategias de aprendizaje y habilidades metafisicas, podría no ser una sorpresa encontrarnos como educadores en el desolador camino de la incertidumbre.

Los sistemas educativos centralizados constituyen otra problemática importante. En estos sistemas las decisiones son tomadas utilizando una estructura piramidal y pretendiendo con esta carencia de liderazgo, cambios en las aulas del sistema escolar por la imposición y la represión. Si el aprendizaje de los estudiantes debe ser profundo, bajo la perspectiva de que los alumnos aprendan a utilizar este conocimiento, más que a recitar una información vacía, los sistemas educativos deberían estar orientados en políticas educativas que faciliten espacios de profundización del conocimiento en el aula. Por el contrario, en muchos países de Latinoamérica los currículum escolares presentan un común denominador; planes y programas de estudio muy cargados de contenido que implícitamente orillan al educador a prestar más importancia a la información y no ha su comprensión y al desarrollo del ser.

Otro aspecto esencial, reside en el desconocimiento o ignorancia voluntaria en la práctica educativa de los docentes, de principios de aprendizaje para el diseño de ambientes que favorezcan la profundización del contenido y el análisis de los sentimientos, ideas y concepciones preconcebidas. Entendemos la profundización como la capacidad del alumno de interiorizar conocimientos teóricos y saberlos aplicar en 
diversidad de situaciones. Claro está, encontramos en ello otro dilema dicotómico: ¿saben los mismos docentes transferir el conocimiento teórico a diversidad de situaciones en sus propios contextos?, es decir, como docentes, ipracticamos lo que profesamos? Los principios que deberían nutrir nuestras decisiones pedagógicas deben centrar el aprendizaje en el estudiante, buscar un aprendizaje significativo, modificar las prácticas de evaluación eminentemente sumativas por otras que recobren su valor de perfeccionamiento humano y crear comunidades de aprendizaje donde la norma general sea el conocimiento y la formación para la vida.

Estos planteamientos resumen una invitación a la aventura de asumir la responsabilidad social de la docencia, con un claro compromiso de impacto en la estructuración una sociedad caracterizada por personas altruistas, críticas, competitivas y más aún, personas capaces de alcanzar su felicidad y la de sus semejantes.

El camino hacia el maestro representa para mí en la actualidad, más que una necesidad académica, un reto personal, pues me ha obligado a enfrentar la resistencia intelectual ocasionada por el desconocimiento y el agobio que provoca la ignorancia consciente. En este esfuerzo, particularmente he tratado de transformar el agobio por regocijo de avance y el fracaso por la comprensión de mis propias limitaciones y las limitaciones de mis estudiantes en clase. He logrado comprender a pesar de haber sido estudiante muchas otras veces, que el aprendizaje a veces duele... y que este dolor solamente puede ser superado con la ayuda de un verdadero maestro. 


\section{REFERENCIAS}

Bransford, J., Brown, A. \& Cocking, R. (2000). How people learn: brain, mind, experience and school, expanded edition. Learning: from speculation to science. (pp. 3-27). Washington: NATIONAL ACADEMY PRESS.

Espiro, S. (2008). Aprendizaje adulto. En: Antología utilizada en el Posgrado de especialización en entornos virtuales del aprendizaje. OEI-Virtual Educa.

Espiro, S. (2008). Aprendizaje. En: Antología utilizada en el Posgrado de especialización en entornos virtuales del aprendizaje. OEI-Virtual Educa.

Hassen, J. (2007). Teoría del conocimiento. México: Editores Mexicanos Unidos.

O'Donohue, J. (2004). El libro de la sabiduria celta. Barcelona: Centro de estudios celtas.

Pausch, R. (2008). La última lección. Barcelona: Random House Mondadori.

Prieto, D. (2008). Planificar para construir futuro. En: Antología utilizada en el Posgrado de especialización en entornos virtuales del aprendizaje. OEI-Virtual Educa. 